This item was submitted to Loughborough's Institutional Repository (https://dspace.lboro.ac.uk/) by the author and is made available under the following Creative Commons Licence conditions.

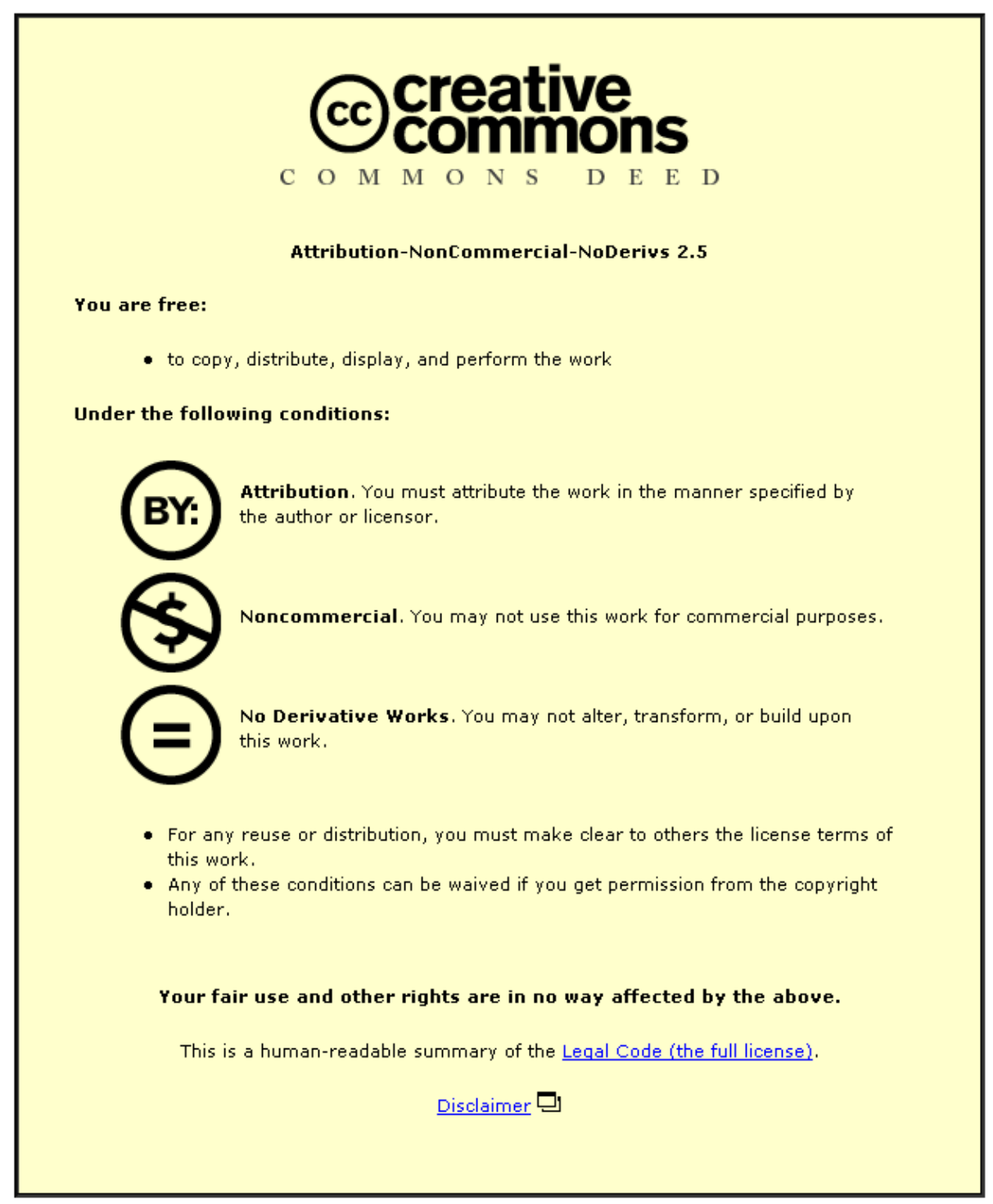

For the full text of this licence, please go to: http://creativecommons.org/licenses/by-nc-nd/2.5/ 


\title{
Referential and Syntactic Approaches to Proving: Case Studies from a Transition-to-Proof Course
}

\author{
Lara Alcock and Keith Weber
}

\begin{abstract}
The goal of this paper is to increase our understanding of different approaches to proving in advanced mathematics. We present two case studies from an interview-based investigation in which students were asked to complete proof-related tasks. The first student consistently took what we call a referential approach toward these tasks, examining examples of the objects to which the mathematical statements referred, and using these to guide reasoning. The second consistently took what we call a syntactic approach toward these tasks, working logically with definitions and proof structures without reference to examples. Both students made substantial progress on each of the tasks, but they exhibited different strengths and experienced different difficulties. In this paper we: demonstrate consistency in these students' approaches across a range of tasks, examine the different strengths and difficulties associated with their approaches to proving, and consider the pedagogical issues raised by these apparent student preferences for reasoning in certain ways.
\end{abstract}

\section{Introduction}

A central unresolved issue in mathematics education is that of how to help students develop their conceptions of proof and ability to write proofs. There has been a considerable amount of research on proof in collegiate mathematics education, much of which has focused on why students cannot construct proofs. Causes of undergraduates' difficulties include possessing different standards of justification than those held by mathematicians (Harel \& Sowder, 1998; Recio \& Godino, 2001), an inability to use, or a lack of appreciation for, definitions in formal mathematics (Alcock \& Simpson, 2002; Moore, 1994; Vinner, 1991), difficulties with formal mathematical notation (Dubinsky, Elterman, \& Gong, 1988; Dubinsky \& Yiparaki, 2000; Selden \& Selden, 1995), a poor understanding of important mathematical concepts (Moore, 1994), and a lack of proving heuristics and strategies (Schoenfeld, 1985; Weber, 2001). For a more complete review of this literature, see Harel and Sowder (2007), Selden and Selden (2007), and Weber (2003). This large body of work has yielded valuable insight into why students cannot construct proofs. However, the important issues of how students can construct proofs and what types of reasoning are desirable while students are producing proofs are still important

The authors wish to thank the students who took part in this research, along with Annie Selden and Adrian Simpson for their valuable comments on earlier drafts. 
open questions. In this paper, we shed some light on these issues by presenting case studies of productive reasoning by two individuals in a transition-to-proof course.

\section{Theoretical Background}

One reason that it is difficult to delineate productive proof processes is the complex nature of proving practice. From one perspective, producing a proof can be viewed as a formal exercise. Griffiths (2000) asserts that "mathematical proof is a formal and logical line of reasoning that begins with a set of axioms and moves through logical steps to a conclusion" (p. 2). It is certainly the case that there are logical rules and formal conventions that arguments must obey to be considered valid proofs by the mathematical community. Furthermore, the use of formalism, precise mathematical language, and logical inference are not only necessary in producing a correct proof; in some cases, they can also be sufficient. In other words, there are cases where one can write proofs solely by starting with definitions and assumptions and logically manipulating these assumptions until one deduces the theorem to be proven. If in doubt, consider the processes that most mathematicians would use to verify trigonometric identities or to establish that the sum of two continuous functions is continuous. ${ }^{1}$ Thus an individual might attempt to write a proof by focusing entirely on logical syntax, without thinking about representations of mathematical concepts. In this case, we say that the individual has taken a syntactic approach to proving (Weber \& Alcock, 2004).

From another perspective, assertions to be proven are not viewed solely as strings of symbols or well-formed formulae. Rather, these assertions can and should be understood as meaningful statements about mathematical concepts, and these concepts can be thought about both in terms of logical symbols and in terms of other intuitive representations such as diagrams and prototypical examples (for instance, see Lakoff and Núñez, 2000). From this perspective, the act of proving a statement can be understood as forming a convincing explanation for why mathematical concepts have the properties ascribed to them by that statement. The formation of this explanation deals not with logical symbols themselves, but with meaningful representations of the mathematical concepts to which the symbols relate. Obviously this reasoning needs to be formalized to meet the conventions of proof agreed upon by the mathematical community, but the formalism of proof in this case is more characteristic of the product, rather than the process, of proving. Such a viewpoint can be seen in the writings of Hanna (1991) who, while stressing the need for proofs to have a legitimate formal and logical structure, refers to this structure as more of a "hygiene factor" and claims mathematicians believe that the importance of a proof lies in the intuitive ideas used to create it. We say that approaches to proving in which individuals make use of meaningful representations of relevant mathematical concepts are semantic or referential approaches to proving (Weber \& Alcock, 2004).

We believe that what we have described above is a false dichotomy. We see deep connections between the two perspectives. To borrow the language of Anna Sfard (1991), syntactic and referential approaches to proving are complementary and represent different sides of the same coin. Examining representations of mathematical objects can indicate logical assertions that are true about those objects and suggest what formal inferences should be drawn in a proof. Conversely, syntactic

\footnotetext{
${ }^{1}$ We are indebted to John Selden for these examples.
} 
logical manipulations of definitions can lead to meaningful assertions that can be used to develop new representations for mathematical objects and modify existing ones (Pinto \& Tall, 1999). However, while syntactic and referential approaches to proving are interrelated and complementary, Tall (1997) and others have argued that some mathematicians tend to predominantly choose one approach over the other. For instance, Tall cites Poincaré (1913), who claimed that Riemann was an intuitive thinker who brought geometric imagery to his aid while Hermite was a logical thinker who never evoked such imagery in mathematical conversation (Pinto \& Tall, 1999; Tall, 1997).

Exploratory studies in mathematics education have indicated that students may also prefer and consistently employ one approach to proving over the other. For instance, Pinto and Tall $(1999,2002)$ investigated the learning trajectories of students in a first real analysis course. They found that some students consistently used visual imagery to make sense of the formal definitions that they were given, while others learned concepts by memorizing definitions and deducing properties of the concepts from the definitions without recourse to imagery. Similarly, Alcock and Simpson $(2004,2005)$ conducted a qualitative study of the behavior of students in a real analysis course. They found that of the 18 students in the study, half regularly displayed multiple indicators of visual reasoning while the other half rarely used visual reasoning in their work. Neither of their studies focused specifically on the activity of proving, but both attest to students' learning and reasoning in a definition-based, proof-oriented advanced mathematical domain.

This paper provides detailed evidence of similar student preferences in the different context of a transition-to-proof course. It contributes theoretically in two ways. First, it allows us to see a contrast between syntactic and referential reasoning in an area in which there are fewer obviously "visual" ways to represent examples of the concepts involved than there are in real analysis. Second, it allows us to examine instances of students' productive reasoning in a transition course. Despite the significance of this course for mathematics students in the United States - it is typically the course in which they are first exposed to rigorous proof (Moore, 1994) - there has been relatively little research on either students' proving processes or their performance after completing this course. Most previous research has focused on skills and concepts that the students lack. Moore (1994), for example, closely followed five students as they progressed through a transition-to-proof course, finding that each of these students had serious difficulties constructing proofs throughout the course. Causes of students' difficulties included their inability to state definitions, not knowing how a proof should begin, inadequate concept images, and an inability or disinclination to generate and use examples. Selden and Selden (2003) presented eight undergraduates in such a course with arguments purported to be proofs and found that these students were unable to differentiate between valid proofs and invalid arguments. The work presented here allows us to see different processes that students use when they are not so hampered by these basic difficulties.

We present data on the reasoning of two students in a transition-to-proof course. Both students were somewhat successful in the course, but one student consistently took a syntactic approach to proving while the other used a referential approach. The goal of this presentation is to: (a) illustrate the processes used by undergraduates while taking a syntactic or referential approach to proving, (b) demonstrate 
that students can be at least somewhat successful in transition-to-proof courses applying either of these approaches, (c) highlight the strengths and weaknesses within each of these approaches by looking at the students' successes and difficulties, and (d) to argue that neither of these approaches should be used exclusively by students and both syntactic and referential approaches to proving are necessary for proving competence.

\section{Research Context}

In this exploratory study, 11 students participated in individual interviews near the end of a one-semester course entitled Introduction to Mathematical Reasoning. In the course, students practiced applying standard proof techniques and were introduced to content on logic, sets, relations, functions, and some elementary group, number, and graph theory. In the study, we observed the students as they completed a collection of proof-oriented tasks. Our overarching goals were to: (a) investigate the degree to which students at this level tended to consider examples of mathematical concepts (either using specific mathematical objects or generic ones) while working on proof-oriented tasks, (b) explore whether there was a connection between students' tendency to use examples and their success in writing proofs, (c) identify purposes for which students used their examples, and (d) explore the difficulties that some students have with using examples to construct proofs.

3.1. Interview Structure. Each participant met individually with the first author of this paper for approximately one hour. In each case, the student was asked to:

(1) Describe their experience in the transition-to-proof course, including their study habits, aspects of the course they found difficult, and their course performance.

(2) Complete two proof production tasks (the material used for specific tasks is reproduced below). They were presented with these tasks one at a time on separate sheets of paper and asked to describe what they were thinking about as they attempted to answer. They worked without assistance from the interviewer until they either completed the task to their own satisfaction or reached a point where they could no longer make progress. The interviewer then enquired about their proving processes, asking about why they had taken specific actions and, if appropriate, about why they found it difficult to proceed. These questions focused on the student's choice of actions and on their conceptions of their own difficulties rather than on conceptual understanding or logical reasoning.

(3) Talk about their answers to two questions on the mid-term examination they had taken the previous week, including any difficulties they had experienced and whether they understood why these had been graded as they had.

(4) Read a proof (see below) and explain this proof; to describe what they would do to explain it to someone who had not yet taken the transitionto-proof course, and to illustrate the proof using examples.

(5) Describe any general strategies that they used when writing and reading proofs, and then to answer more specific questions about whether they focused on examples or "rules" during such work. 
The aim of having the interview structured in this way was to gather information about students' use of examples in a variety of different proof-related contexts. We included both proof production and proof reading tasks since these might reasonably be expected to elicit different behaviors. We included the examination questions in order to allow discussion of at least some mathematics completed outside the interview and without the requirement to think aloud. Again, this would help us to investigate whether students were consistent in their use (or otherwise) of examples in their reasoning. Finally, we deliberately postponed asking the participants for reflections upon their own use of examples until the end of the interview, in order to minimize the chance that they would be influenced by this line of questioning while they were addressing the mathematical tasks.

In this paper we present data from the last four sections of the interviews. Reproduced below are the two proof production tasks, one examination question, and the statement for which the participants were asked to read a proof (the proof itself appears in the appendix).

Relation task (proof-production)

Let $D$ be a set. Define a relation $\approx$ on functions with domain $D$ as follows. $f \approx g$ if and only if there exists $x \in D$ such that $f(x)=g(x)$. Prove or disprove that this is an equivalence relation.

Function task (proof-production)

Definitions:

A function $f: \mathbf{R} \rightarrow \mathbf{R}$ is said to be increasing if and only if for all $x, y \in \mathbf{R},(x>y$ implies $f(x)>f(y))$.

A function $f: \mathbf{R} \rightarrow \mathbf{R}$ is said to have a global maximum at a real number $c$ if and only if for all $x \in \mathbf{R}$ such that $x \neq c$, $f(x)<f(c)$.

Suppose $f$ is an increasing function. Prove that there is no real number $c$ that is a global maximum for $f$.

Examination question

Let $S$ be a set with at least two elements so that there are subsets other than $S$ itself and the empty set. Consider the power set of $S, P(S)$, that is, the set of all subsets of $S$, under the inclusion relation $\subseteq$.

Determine whether or not this relation on the power set is: i) reflexive, ii) symmetric, iii) antisymmetric or iv) transitive. Justify your claims.

Proof-reading task

Definition:

A function $f: \mathbf{R} \rightarrow \mathbf{R}$ is said to be increasing if and only if for all $x, y \in \mathbf{R},(x>y$ implies $f(x)>f(y))$.

Theorem:

Suppose $f$ and $g$ are increasing functions. Suppose that $f(0)=$ $g(0)=0$. Prove that for all $x \in \mathbf{R}, f(x) \cdot g(x) \geq 0$.

All of these tasks involve producing or reading proofs that involve general assertions about classes of mathematical objects with certain properties. The relation task involves a general set and (possibly) a general element within that set, though 
the relation itself is fully specified. The function task involves a general increasing function. The examination question involves a general set with certain simple properties, and once again a fully specified relation. The proof-reading question involves two general increasing functions. In all cases, definitions of relevant properties of these general objects are either provided or (in the case of the examination question) are expected to be familiar to the student. Hence, in all cases, an individual may reasonably approach the question either by working syntactically with the appropriate definitions or by examining particular instances of the general objects discussed.

The reader will note that we did not include any questions of the types "prove that this statement is false," "decide whether this statement is true or false" or "give a counterexample." This was a deliberate choice, made in line with our aims for the interview as stated above. Where work on all of the included tasks could reasonably begin with either a syntactic or a referential approach, we were concerned that any task that strongly suggested consideration of (counter)examples might affect the participants' inclination to talk about examples in their other reasoning or in their reflection upon this. We are interested in responses to such questions and in the issue of counterexamples and their role in mathematical learning and reasoning, so we will return to this briefly in our discussion at the end of the paper. However, we judged that it was not appropriate to include a question of this type in this particular study.

We note that it remains possible that any of our findings about example-use in interacting with proofs may be an artifact of the particular questions we asked about the particular types of mathematical object involved. This is one limitation of this study, and indeed elsewhere the first author has speculated that the degree to which an individual tends to refer to object-like visual representations may depend upon the accessibility of these within subject matter (Alcock \& Simpson, 2002). However, these interview tasks involve a variety of objects. These range from real functions, for which all students had long experience of interacting with graphical representations, through inclusion relations, which were newer but may also be represented visually in a number of straightforward ways, to equivalence relations, which the students were less likely to think of as "objects" and for which they were certainly unlikely to have assimilated any substantial non-algebraic imagery. We therefore suggest that this range of tasks went some way towards allowing each participant to display a range of strategies if they were so inclined.

3.2. Analysis. The interviews were transcribed and the transcripts were analyzed by the two authors. First, each author independently read through two transcripts and identified episodes in which the student used an example. Codings were compared and any disagreements were discussed. Once it was clear that there was a high level of agreement about these codings, we then constructed a scheme for analysis in which each author would independently:

- Identify the interview context in which the example was used.

- Note whether the use was prompted by an interviewer question.

- Characterize the purpose for which the example was used.

- Describe the example.

- Note any difficulties with using the example. 
Through this analysis it became clear that some students took a consistently referential approach to proving. Six students used examples in their attempts to answer multiple questions, used their examples for multiple purposes, and made reflective comments indicating that they regularly made use of examples in their proof-writing. Four students took a consistently syntactic approach to proving. These students did not refer to an example on a single task that we provided for them, and made no comments that they regularly made use of examples in their usual work for the course. There was one remaining student who was less consistent in her behavior. A more complete discussion of all the students' behavior can be found in Weber, Alcock, \& Radu (2005).

3.3. Case Studies. The distinction between referential and syntactic approaches was particularly sharp in the more successful students, two of whom provide the case studies that form the remainder of this paper. These two students, Brad and Carla, attained an A- and an A respectively in the midterm examinations that they had taken the week prior to the interviews (grades are on the scale A, B, $\mathrm{C}, \mathrm{D}, \mathrm{F}$, where $\mathrm{D}$ and $\mathrm{F}$ are considered failing grades). In their interviews, neither student was perfectly productive or efficient in their proof attempts, but both were coherent and both took actions that were mathematically sensible and likely to lead to progress. Brad, however, spontaneously referred to examples in response to all of the interview tasks, while Carla never did so.

We note that this occurred despite the fact that they had attended the same class and had been exposed to the same lectures, the same homework assignments, and the same earlier examination. We do not have information on the students' previous experiences with proof (though these are unlikely to be extensive) or mathematics in general, so we cannot comment on how these tendencies may have arisen. For instance, it is conceivable that Brad at some point had a mathematics teacher who made a point of referring to examples, that Carla had a teacher who stressed systematic writing, or that either student simply had one success early on in this particular course with their preferred strategy and so stuck to it. However, whatever the source of these tendencies, we can say that they appear to have resulted in these two students interacting very differently with the material as it was presented to them in their transition course and in the interview. We return to implications of this finding in our concluding discussion.

In addition to addressing the interview tasks competently, Brad and Carla were both articulate in reflecting upon their own strategies, and so provide good material for us to see how referential and syntactic approaches have distinct advantages and disadvantages. They also provide an opportunity for us to recognize that students with nominally similar levels of attainment might be achieving this in strikingly different ways, and that those who are successful might become even more so if we can recognize which of their skills they are using well, and which would benefit from further development. At the end of the paper we will also consider ways in

which study of the good-but-imperfect performance of these students might inform our teaching of those who are doing less well in transition courses.

\section{Referential Approach: Brad}

Brad used examples in his reasoning consistently across all of the interview tasks, and the examples he chose were appropriate in each context. His reflective comments indicated that this referential approach was something he used regularly 
and often deliberately. His behavior and his reflections indicated that it was natural for him to use examples specifically to:

(1) Get an initial understanding or grasp of the concepts in a given question.

(2) Illustrate a proof to someone else.

(3) Decide on a type of proof to use.

(4) Fall back on for more ideas when he could not decide how to proceed.

Illustrations of the first and second of these are provided in Section 4.1, where we see that his referential approach seemed to serve Brad reasonably well in that it afforded him a sense of understanding of a given mathematical situation. Illustrations of the third and fourth are provided in Section 4.2, in which we examine in more detail the ways in which Brad's referential approach helped him and the ways in which it turned out to be less effective. In particular, though it allowed him to decide on a type of proof to pursue, and provided him with a way to search for more ideas, it did not afford him the ability to use this insight to write a full and correct general argument. Finally, in Section 4.3 we provide a short discussion to conclude this section, including further illustrations of Brad's descriptions of his own strategies.

4.1. Strength: Using Examples to Understand Concepts or Questions. Brad spontaneously invoked examples as a first response to all of the tasks discussed in the interview. On reading the relation task he made an initial comment that he was "trying to think what the question's asking," then announced,

B: Alright, I'm just going to like write out some examples. To try and. . like, set a $D$. And then...yes, write out a function or two. I don't know if that's going to help me.

He wrote the following on his paper:

$$
D=1,3,5 \quad f(x)=x^{2} \quad g(x)=x
$$

When asked later why he had done this, he said,

$B$ : Um, I guess it just... gives you something concrete [...] Because this is really general. And you can't really put your hands on this. You know I can't like, get a grasp of it.

B: Um, I think just to get an idea of what the relation was. [...] When we're given a relation, a lot of times it's a little more like, you know "greater than," you know or "equal to." Or something like that. Or... it's easier. Like this is kind of, a little like harder to see.

Similarly, on reading the function task, Brad again commented that he was trying to understand the question, and stated:

$B$ : And I'm going to take an example to make sure I'm doing it right.

He wrote the following, along with a small sketch of a graph for $f(x)=x$, on his paper:

$$
f(x)=x \quad x=2 \quad f(2)=2 \quad y=3 \quad f(3)=3
$$

On completion of the question, he once again indicated that he was using examples to grasp the concepts:

B: I didn't, I never heard of a global maximum. I don't think we learned about increasing, but I'm not sure. I don't remember learning about it. So I wanted to teach it to myself first. And, I want to teach myself by examples, you know. And I was kind of starting to understand a bit 
more when I was trying to, in trying to grasp - I grasped increasing, it seemed like, okay. But then I was trying to grasp the global max.

Brad's written examination solutions and his interview comments about them also followed this pattern. In response to the examination question, Brad's first line of writing read:

$$
S=\{a, b\} \quad P(S)=\{\{a\},\{b\},\{a, b\}\}
$$

When asked in the interview to talk through his thinking while working on this question, Brad explained:

$B:$... we hadn't dealt with power sets in a while, so it kind of threw me off at first. But then I was like okay, it's not that hard. Calm down.

I: Okay.

$B$ : So then, I set up a set $S$, and then I...um... I told what the power set was. So I could kind of look at... like I could see the reflex - the relations? Like, for myself.

Finally, after reading the proof-reading question, Brad was asked how he would explain the proof to someone who had not taken the course. He responded,

$B$ : Um ... I would most likely try to relate it to something that they come and do understand. Um, maybe ah, I'd probably also like draw a graph. Like first, introduce the idea of an increasing function. And then show, you know, you know, $g$ of... that ah, if $x$ is greater than $y, f$ of $x$ must be greater than $y$, by the terms of the increasing function.

When asked to show the interviewer what he would do, he decided to "take the easiest $f$ of $x$ is equal to $x$," and also looked at particular numbers in order to illustrate the increasing property:

$B$ : So we'll take $a$ to be 1 so $b$ must be greater than that, $b$ to be 2 . Um... and, so, since $f$ of - oh, I'd probably show them that $f$ of $a$ is equal to $a$. And $f$ of $b$ is equal to $b$. From $f$ of $x$, or whatever. [...] So then, ah... $f$ of $a$, is also 1 and $f$ of $b$ is also equal to $1-$ ah, 2, for all cases. And then show them on the graph, you know 1,1 and 2,2.

Throughout the interview it appeared that it was important for Brad in his mathematical work to feel that he could "grasp" or "see" the concepts in any given question, and that by examining examples he could achieve this to his own satisfaction.

4.2. Difficulties: Translating Understanding into Proof. In fact, Brad showed himself to be adept at choosing appropriate examples, at manipulating these as entities without losing track of their component parts and at using them to outline formal arguments. We will see this in this section. However, we will also see that although his insights were usually correct, he struggled to translate these into acceptable mathematical arguments. In this section we focus on the relation and function questions because these are the ones where Brad attempted to construct proofs of his own.

After deciding that he was satisfied with his example for the relation question, and recalling the required properties for the relation to be an equivalence relation, Brad reasoned about reflexivity and symmetry thus:

$B$ : So... so okay if it's reflexive, then... $f$ of $x$ should be equal to $f$ of $x$. Or there should be $x$ in $D$ with, so that $f$ of $x$ is equal to $f$ of $x$. Okay. 
That's all I'm going to say! Laughs. And... that's true. Because 1 is equal to 1 . Symmetric, is um... $x-f$ implies $-f$ is related to $g$ implies $g$ is related to $f$. So... so this is really the $g$, there's an $x$ in $D$ such that $f$ of $x$ is equal to $g$ of $x . g$ is related to $x-\mathrm{ah}, f$, when there's a $x$ in $D$ such that $g$ of $x$ is related to $f$ of $x$. Pause. So... implies that $g$ of. ...yes. Because if... because $x$ [is] $1, f$ of $x$ is equal to $g$ of $x$, then the same $x$ in $D$ that $g$ of $x$ must be equal to $f$ of $x$.

We note that Brad spoke about $f$ and $g$ as though these stood for general functions, but occasionally referred to his example as if to confirm his thinking. We note also that throughout his work (unlike many of the weaker students in the study), Brad apparently had no problem maintaining control of the objects in the question: he correctly considered reflexivity, symmetry and transitivity as possible properties of the defined relation, without becoming confused about the different types of objects involved (an arbitrary set, elements of that set, functions on that set and a relation between these) or about the way in which the notation represents these.

While working on this and the rest of the question, Brad made some brief notes, but did not write using complete mathematical sentences. When he had finished, the interviewer asked him whether he would write anything else if he were to hand this in for homework, and what this would be. Brad elected to demonstrate for reflexivity, "since it's the easiest part," and he wrote the following:

Reflexive is when $f \approx f$.

$f \approx f$ assuming there exist an $x \in D$ such that $f(x)=f(x)$.

Since $f(x)=f(x)$ the relation reflexive.

A generous interpretation might say that he excludes (sensibly) the possibility that $D$ is empty by assuming that there is an $x$ in $D$, and that for this $x$ we will naturally have $f(x)=f(x)$. However, even if his thinking is as clear as this, the clarity is lost in his written work, in which poor use of syntax means that there is ambiguity regarding what is assumed and what is inferred. The interviewer did not challenge what Brad had written and he did not appear aware of this ambiguity.

In his work on the relation task, Brad was able to provide written answers and feel that he had answered the question. In his work on the function task, however, this was not the case, and we can see more clearly the problems encountered while trying to apply a referential strategy during proof construction. After writing down his initial example Brad continued exploring the defined concepts. He wondered aloud, "Can $f$ of $c$ be repeated?" and sketched a sine curve with two local maxima marked, before rejecting this idea. He then talked quietly to himself for a moment before suggesting an overall proof tactic.

B: I think we can do this by contradiction. Assume that... assume that um... if $f$ is an increasing function then $c \ldots$ ah...then there is... a $c$ ? For which there is a max. And then prove that that can't happen. And then, so that'll prove it.

He began to work on this idea, but found it difficult to make progress.

$B$ : Alright so, if there is... a global max... writing, mumbling... $f$ of $c$ is greater than both $f$ of $x$ and $f$ of $y$. I'm trying to think like, you could um, I'm taking the, I'm trying to think that is $c$, and see if I keep repeating the process, like make um, how I could show that if you, show 
that there's another $x$, plus, like $x$ greater than, like if $x$ is greater than $y, f$ of $x$ is greater than $f$ of $y$, then there's a number greater than $x$, that'll show that it's greater than - that number's greater than $x$, and then that's like, keeps going, and going and going. But I don't know how...

After this struggle, he returned to consideration of his graph of a generic increasing function:

$B$ : I'm just trying to see it by looking at the graph. How I can relate it. Like, the two terms interrelate. Why. . because I can't even see - I want to know why, there can't be one [...] like know why it can't be and then try to prove.

He appeared ready to give up shortly after this, and when the interviewer asked whether he could talk through his thinking, he said,

$B$ : Alright. I'm thinking that in the definition of increasing, there's never going to be one number that's the greatest. There's always going to be like, a number greater than $x$. Because it's, because it's increasing. So there's always going to be some number greater than the last. So if $x$ is greater than - that's what I assumed here. $x$ is greater than $y$, then there's going to be some $x$ plus 1 , that is going to be greater than $y$ plus 1 , so that $f$ of $x$ plus 1 is going to be greater than $f$ of $y$ plus 1 . Or something like that. Where like, it's just going to change.... So then, there can't be some number, you know that... if it's increasing there can't be some number that's greater than all of them. Or, some $f$ of $c$.

In our view Brad seemed to have some reasonable idea about the fact that for any number in the domain, one can always take a greater number, whose image under the function will be greater than that of the original. However, he did not seem to have good control over the way in which the definitions, and in particular the variables $x, y$ and $c$, could be used to express this argument. We think it is worth noting that Brad did not attempt to link these variables explicitly to his sketched graph by labeling it in any way.

4.3. Discussion: Brad on His Reasoning Strategies. Overall, Brad seemed able to choose appropriate examples, both specific and generic, and to work with these with some fluency. However, he did not seem to use his examples to effectively guide his manipulation of the symbolic notation at the detailed level. In fact, his own reflective comments on his general strategies for writing a proof suggest that he was not trying to do this, relying instead on his knowledge of standard types of proof to provide structure:

$B$ : I start out by forming an example to, you know, get a strong grasp of what they're asking me. And then, ah, probably play around with like, maybe do a few examples, so I can see what it's - actually maybe how I could prove it, which method of proof I should use. And then once I find a method, proceed from there and... just remembering, just... because it seems like in all the different types of proofs we've done, there's always some kind of structure. So once I get to know what the proof is, I feel more comfortable because I guess that's the hardest part of, of proof. You know, is figuring out which kind of proof to use, because then you can structure it the way you've normally done it before. 
This use of examples for specific purposes at specific junctures in his work was further confirmed by his answers to more explicit questions about whether he tended to think about examples, rules or a combination of the two when writing and reading proofs.

B: [about writing proofs] Probably a combination but more the rules. I probably use the examples just as a. . . um, more specific, so I can get like exactly what it's telling me. Or more, probably, based on the question. And then with the proof um, try and like base it on, like on the properties or...

B: [about reading proofs] More examples, probably. Just so that like... I always find that, whenever I, I guess like the more I write down, the more I can see... the more clear I'll be. [... . And even - even during the proof, like maybe refer back to it, I guess like the last question but... you know I could try to use - if I got stuck somewhere... in the proof, where I'm like, I don't know where to go from here, look back at - I guess I do look back at the examples I used, I guess to see where I could go from there.

At the end of this paper we will offer further discussion of the range of purposes for which students use examples in proof-related activity and the way in which they relate these to more syntactic work.

\section{Syntactic Approach: Carla}

In marked contrast with Brad, Carla did not spontaneously use examples in response to any of the interview tasks. Instead, she focused on the syntax and form of the given definitions, questions, and written proof. Her reflective comments indicated that this was a consistent and deliberate approach, and is demonstrated in Section 5.1. Both Carla's reasoning in the interview and her reflective comments indicated that she was often successfully able to answer questions using this syntactic strategy; further, she appeared to have fewer difficulties than Brad in constructing written arguments. However, where Brad's referential reasoning gave him security in the correctness of his own answers, Carla sometimes expressed doubts about her arguments. We illustrate this in Section 5.2, where we also note that although Carla could generate examples when asked to do so, this did not seem to occur to her as a possible approach, and she expressed doubts about the idea of using examples as a tool for mathematical reasoning. Finally, in Section 5.3 we discuss Carla's comments about her strategies in general.

5.1. Strengths: Using Syntax and Form to Construct Arguments. The first notable feature of Carla's responses to the relation and function tasks was that she began speaking and writing immediately after reading the question. For the relation task she listed the properties of an equivalence relation, and went on to draw a quick conclusion.

$C$ : Oh... okay. It's transitive, symmetric, and reflexive. [Writing.] So to prove that it's transitive... um... pause... if $x$ is in $D, f$ of $x$ is. . equal to $g$ of $x$. $f$ of $x$ is equal to $f$ of $x$, so $f$ is related to $g$. So it's reflexive...um...symmetric is... if $f$ is related to $g$, then... $f$ of $x$ is equal to $g$ of $x$, so $g$ is related to $f$ as well...so...symmetric. And transitive is... $f$ is related to $g$, that means $f$ of $x$ is equal to $g$ of $x$, 
and $g$ is related to... a I guess... so $g$ of $x$ is equal to $a$ of $x$. So it's transitive as well. So... yes. It's an equivalence relation.

Notice that Carla did not give due consideration to the existential quantifier in the definition of the relation (Brad made a similar error in this part of the question); the interviewer did not attempt to draw her attention to this. Like Brad, Carla had made short notes, and the interviewer asked whether she would write anything else if she were going to hand this in for homework. Carla said yes and elected to provide an answer for symmetric. She wrote the following:

$$
\begin{aligned}
& \text { Symmetric YES if } f \approx g \text {, then } f(x)=g(x) \\
& \text { if } f(x)=g(x) \text { then } g(x)=f(x) \\
& \text { thus } g \approx f, \text { so if } f \approx g, \text { then } g \approx f \text { thus it is symmetric. }
\end{aligned}
$$

We note that the ambiguity in quantification persisted, but that her written answer is more clearly structured than that provided by Brad.

Carla's response to the function task began in a similar way, with reading of the question followed by immediate writing.

$C$ : So...I'm thinking the way to prove this is using contradiction. So, I would start out by assuming... there exists... a $c$... for which [writ$i n g] \ldots f$ of $x$ is less than $f$ of $c$, when $x$ is not equal to $c$. Okay. [Pause.] So now I'm trying to use the definition of increasing function to prove that, this cannot be. Um... so there exists a real number for which $f$ of $x$ is less than $f$ of $c$, for all $x \ldots$ and there's... $f \ldots$ is an increasing function... for... all $x \ldots$ [writing] $\ldots y$ in $\mathbf{R}, x$ greater than $y$ implies $f$ of $x$ greater than $f$ of $y$. Mm... pause... I guess what I'm trying to show is if $x$ is in reals, and they are infinite... for all $x \ldots$ there will be... some function $f$ of $c$ greater than $f$ of $x$. [Long pause.] So... there exists... an element... in $\mathbf{R} \ldots$. [writing] .. greater than $c$. Um... for $x \ldots$ because... $f$ is an increasing function... $f$ of $x$ will be greater than $f$ of $c$. Um... a contradiction... so that... there is no $c$ for which $f$ of $c$ is greater than $f$ of $x$... for all $x$.

Carla's comments when asked to reflect upon this task provide evidence that her approach was syntactic throughout. When asked what immediately made her decide to prove by contradiction, Carla answered to the effect that she had used the form of statement to decide upon an appropriate proof structure.

$C$ : Because, in class, whenever we have some statement which says, "There is...no such number," or "There exists no such number," then we assume there is, such number. And then we go on to prove that that would cause a contradiction, thus, it doesn't exist. So it was just, something. . . automatically ingrained, when I see those couple of words, I think contradiction.

The interviewer then commented that Carla had appeared to be stuck for a short time, and in response Carla described having to think about the problem once she had systematically begun her answer.

$C$ : Well the first line kind of came from me, you know just recognizing those first couple of words and recognizing the format it should be starting off with. Because contradiction, so assume this, this number exists. And then I was thinking, I should take what's previously given, that it's an increasing function. So I wrote that down. And once I was done 
with that, systematic part, I kind of needed then to think about, how actually... actually think about the problem.

Although one might imagine that this "actually thinking about the problem" would involve some other representation, further probing revealed only that she was thinking about what a written proof would look like. Indeed, later in the interview Carla responded to direct questions by stating clearly that she was not referring to examples while working on this task.

I: Did you have any sort of picture in your head for this one?

$C$ : No, no...not really. I mean I know what a global maximum is from calculus... I mean I've done these sort of things so many times. But I didn't imagine any, any sort of function. Something that would have a maximum. [...] Really... I guess I did it very systematically and theoretically, because I just stepped - this is the rule, and do it through.

I: Okay. Yes. You see [...] one thing I thought you might be doing is trying to create some kind of picture or trying to imagine how that would work with a particular example. And that appears to be not what you were doing in that case...

$C$ : No. No. I just, I just tried to see if it was, it was connected. Just theoretically, if the words made sense. If one thing implied the other.

Carla's responses to the examination question and the proof-reading question provided evidence that she used a syntactic approach in other situations. The first lines of Carla's response to the examination question read:

$S$ set with at least two elements

$P(S)$ under $\subseteq \quad x$ is element of $P(S)$, so $x \subseteq S$ $y$ is element of $P(S)$, so $y \subseteq S$

We note that where Brad wrote down a generic set and its power set, Carla wrote down and elaborated a list of the given conditions of the problem. When asked about this question, Carla commented "Basically the format I used was like the same one I used up here," referring to the earlier interview question. When asked to talk through her thought processes, she gave a general description of how she thinks about such questions:

$C$ : Okay. So, I just think when, when something is reflexive I think, so, there's this one element related to itself, and, does that relation hold true?

I: Okay.

$C$ : I guess that was how I think about it. I just pick two elements, if it's for symmetry, or antisymmetry I pick two elements, and say if they're related, then what does that mean, and... from then on can I conclude some next relationship between them.

For the proof-reading task, when Carla was asked how she would explain the proof to someone who had not taken the course, she did not refer to any examples and apparently did not feel the need to add much further explanation. Indeed, when specifically asked to illustrate the proof using examples, Carla expressed uncertainty about what she was being asked to do:

C: Just... what do you mean? [Hesitation.] What type of examples?

We explore the apparent impact of Carla's overall lack of example use in the next section. 
5.2. Difficulties: Absence of a Sense of Understanding. The most striking illustration of the disadvantages of Carla's syntactic approach occurred after her initial response to the function task. Despite her competent attempt, which stood out as unusually efficient and complete compared with those of most student participants, she commented on her proof that "it seems a bit flaky." When asked why, she said,

$C$ : I don't know, it just doesn't make sense for me. It, it feels like, I just, it's just proved systematically, without being able to imagine what's going on. So that's why it feels flaky.

As we noted before, evidence throughout the interview indicated that although she talked about "imagining what is going on," she did not at any point consider examples or generic images of functions. This is not to say that Carla was unable to generate examples, however. This is best illustrated by her eventual response to the proof-reading question. As noted at the end of the preceding section, she initially seemed thrown by the request to generate illustrative examples. However, with an opportunity to think further she went on to address the question without apparent difficulty or errors.

I: Well, whatever you think would be appropriate, really. Take your time if you want to think about it, and do write anything on the paper if you want to.

$C$ : Okay.... I guess I would give two simple functions, $f$ and $g$ that were both increasing.

I: Okay.

$C$ : And, such that...I guess... writing... $f$ of $x$, equal to $x \ldots$ and...g of $x$ is equal to... some other increasing function. Like... kind of like $x$ cubed.

Like Brad, Carla stated further that she would illustrate the increasing property by using particular elements of the domain, and showed how she would do this when invited to do so by the interviewer.

$C$ : And then... I would just give... well first I would explain why they were increasing, and just give examples of different...x s. If I were to explain it...

I: Do you want to do that for me now?

$C$ : Oh, okay. Example... for why it's an increasing function. Just take, I guess... $x_{1}$ is equal to $1, x_{2}$ is equal to $2 . f$ of $x$ would be... $x$ and... $f$ of $x_{2}$ would be 2 and so it's... $f$ of $x_{2}$ is greater than $f$ of $x_{1}$. Because $x_{2}$ is greater than 1 , they are increasing. So I would say that first. Then I would go on to, for each of these three cases, I would give an example for, for instance, for some $x$, say $x$ is negative $2 \ldots$ that's for the second case...

Here we observe that although Carla's language did not perfectly capture the logical relationships between what was assumed and what was demonstrated or illustrated, she did introduce appropriate examples of both functions and domain elements, and she did coordinate general statements and particular objects correctly throughout.

In fact, although the idea of using examples in reasoning seemed rather alien to Carla in relation to the tasks discussed in the interview, it turned out in the final 
discussion that she did refer to examples when reading proofs, and gave reasons similar to those given by Brad.

$C$ : I think about examples, when I read proofs. Because, it just tends to clarify it better.

I: Okay. And, how do you decide what examples to think about, in that case?

$C:$ Um...I just think any random examples, or different cases if there are only a set number of cases. You know I think of one for each case. But, other than that, any example. The simplest ones, usually. Laughs.

I: Right. Can you give me an illustration of the kind of thing you would look at in a certain kind of problem?

$C$ : Well, sometimes, like in graph theory, even when I do the proofs, I, I sometimes don't even understand what is supposed to be proven. I understand the assumptions, and then from the assumptions I have to draw an example for myself, of some simple graph that has this property. And then... have to prove to myself that that would be true, in that case. And then I go on from there, to understand that proof.

However, when asked whether she had ever tried a similar strategy when writing proofs, Carla expressed discomfort with the idea of using examples as a basis for constructing general arguments.

$C$ : Even if I have convinced myself that that proof would be true, and it would happen in certain examples, it wouldn't help me in writing out the proof itself. Because it has to hold for all graphs, and... I don't know how to explain it. I have trouble... generalizing graphs. As a whole. So those examples wouldn't work in that case.

Indeed, Carla expressed a broader discomfort with the use of examples in proving, including problems she had encountered in using counterexamples in disproof.

$C$ : I could never grasp the, just concept of giving a simple counterexample, any old thing. And those were usually the easiest problems on the exam. And I would always get zeros on them. Because I tried to disprove it in a general manner. And, I guess I'm just not, I don't trust examples, but...

It is not clear whether Carla had been over-influenced by the maxim "you can't prove by example" or whether she simply found herself unable to generate a proof based on observation of an example.

5.3. Discussion: Carla on Her Reasoning Strategies. Overall Carla took a consistently syntactic approach to all the tasks discussed in the interview, and she both confirmed and elaborated upon this in her later reflective comments. When asked about any general strategies she had for writing proofs, she said,

$C$ : Um, I just start with a claim... I usually don't have anything in my head beforehand. I start off with what I know, and then I assume, what they're talking about, that I should use, in that case. And then I just try to work off of there. And I try to imagine what my goal is, and kind of work from both sides, to the center. 
When asked more specifically about the first things she would do, she stated that she "thinks of a method to use" and went on to explain how she identified an appropriate one:

$C$ : If it says, if it's something that has to be proven for all... numbers in such a set, then I use induction. And...for instance, if uniqueness is supposed to be proven, I always assume there's two different numbers that produce the same result. Or something to that extent. And use contradiction. Or, for there exists no number such that, I say yes, assume there is and then use contradiction.

This basic strategy still stood when she did not immediately know which technique to use.

$C$ : Well I would try out just different ones and see which one gets me the farthest. [...] We don't really know many methods, so it's not that difficult, to get one right.

This last comment indicates that a syntactic approach afforded Carla the ability to answer most of the questions she encountered in the transition course. As we have seen, what it did not appear to afford her was a sense of meaningful understanding of her answers, unlike that which Brad appeared to obtain by reference to examples. Interestingly, as we saw in the previous section, the use of examples to gain understanding of a situation was not alien or inaccessible to Carla. However, in her case we can see an absence of two-way links between examples and what she knew about proof structures, and a very restricted use of examples in proof-related activity.

\section{Discussion}

6.1. Summary: Referential and Syntactic Approaches to Proving. Compared with the majority of the interview participants, both Brad and Carla scored well in their course examinations and made substantial progress on the interview tasks. Further, at times in the interview they made similar errors relative to both conceptual correctness and the precise use of mathematical language to express logical relationships. However, we believe it is clear that they approached these proof-related tasks very differently, and that they exhibited different strengths and experienced different difficulties. Below, we review these approaches and difficulties before considering the pedagogical implications of this research.

Brad took a referential approach, referring to examples of the statements he was considering. Sometimes these referents were represented in an obviously "visual" way, as in his discussion of graphs while working on the function task and the proofreading task. Sometimes they were represented using strings of symbols signifying mathematical objects, as in the written sets he introduced while working on the tasks involving relations. Sometimes he examined particular examples, such as the function $f(x)=x$ or the set $D=\{1,3,5\}$. Sometimes he examined more "generic" examples, such as the set $\{a, b\}$ and its power set. However, in all cases he treated the statements as being about mathematical objects and their properties, and he examined examples of these objects regularly in order to address the questions. This referential approach afforded Brad a strong sense of meaningful understanding he appeared satisfied that by considering example objects he could understand the meanings of concept terms and gain insight into why a statement should be true 
(for more on this idea, see Alcock \& Simpson, 2004). Both the proof production tasks and Brad's own reflections suggest that the approach also afforded him a means of deciding on appropriate proof structures for given statements. What it did not seem to afford him was a consistent ability to coordinate the details of a general argument. In the interview, Brad sometimes gave reasonable examplebased arguments but struggled to produce proofs in terms of the relevant formal definitions. His reflective comments suggest that a possible reason for this is that he treated understanding (via examples) and proving (via familiar proof formats) as somewhat separate parts of his activity.

Carla, on the other hand, took a syntactic approach. She did not consider the referents of the statements unless prompted to do so. Instead, she took cues from the linguistic form of the statements she was considering, using these to decide on an appropriate proof structure. She used known or given definitions to systematically write down what was to be assumed and what was to be proved, and searched for logical inferences she could make that would lead from one to the other. She explained her own reasoning in these terms, and did not appear to feel a need to add anything beyond this type of explanation when talking about a provided proof. This syntactic approach served her well in producing written answers to the interview tasks, and her reflective comments suggest that she found it generally effective in the course as a whole. However, Carla's comments also indicated that she was sometimes able to produce such work without being able to "imagine what is going on," and that this could make her hesitant about her answers even when from an observer's point of view these were full and correct (for more on this idea, see Raman, 2003). Moreover, while Carla saw the value of looking at examples in order to understand a statement, she commented that she had struggled with tasks requiring the generation of counterexamples, and did not see how the use of examples might help her in constructing a general proof. Finally, while one may be able to use a syntactic approach to construct a fair number of proofs in the conceptually limited domains that are explored in a transition-to-proof course, we question whether one can construct a wide range of proofs in a more sophisticated, conceptually rich mathematical domain by relying exclusively on a syntactic approach to proving (see also Weber, 2001, and Weber \& Alcock, 2004).

6.2. Theoretical Discussion. The issue of how referential and syntactic strategies contribute to effective mathematical reasoning is both interesting and complex. Certainly a successful mathematician will be able to use both, switching from one to the other in response to the perceived demands of the situation (Alcock, 2008). For instance, a mathematician who is uncertain about the truth of a statement is likely to examine examples in a more or less systematic counterexample search, whereas one who is certain of a result may proceed directly to working syntactically with appropriate definitions (Inglis, Mejia-Ramos, \& Simpson, 2007).

However, as we noted in the introduction, it has been suggested that some experienced and famous mathematicians exhibit tendencies to use one or the other strategy preferentially. Our own experience of discussing these issues in a variety of presentations with both mathematicians and mathematics educators suggests that such tendencies are widespread, and this study, along with others (Alcock \& Simpson, 2004, 2005; Pinto \& Tall 1999, 2002), indicates that many students exhibit similar tendencies at the transition-to-proof level. 
This leads naturally to the question of the origins of these tendencies. We are often asked whether we suppose that they are innate, or whether we think that they are learned through earlier mathematical experiences, in school or elsewhere. We acknowledge that this is a fascinating question, but we also believe it is highly intractable - it isn't feasible to trace even one individual's entire mathematical development, much less to compare many such paths through mathematical learning. We are more interested in the more practical question that may be stated as follows: Given that right now we have students in transition courses who display tendencies to favor referential or syntactic strategies in their reasoning, what should we do about it? We offer some comments on this in the final section.

6.3. Pedagogical Implications. As we have described, Brad's and Carla's respective approaches were consistent across tasks that asked for proof production, for proof explanation, and for description of reasoning undertaken in an examination context. They were also consistent with the students' reflections on the reasoning strategies they generally employed in their transition-to-proof course. If we accept that these are instances of somewhat common tendencies, then this provides a differentiated backdrop against which we can offer some comments on what it is desirable for students to learn in transition-to-proof courses, and how instruction might work toward this in the cases of more and less successful students.

First, it could be argued that Carla's syntactic approach is "better" in the important respect that it affords more success in producing written proofs and fewer errors of logical expression within these. As mathematicians, we certainly value the systematicity of such a syntactic approach and our assessment methods reflect the fact that our primary goal is that students should learn to produce correct written proofs. However, as suggested in the theoretical background, the syntactic approach corresponds to only one view of proof. The skills used in proving syntactically are only a subset of those that we might wish a student to develop. Others pertain to interpreting mathematical statements as giving meaningful information about mathematical objects and using other representations of these objects to guide proving activity. In Weber \& Alcock (2004), we argue that use of such skills contributes significantly to mathematicians' ability to construct proofs, and in Alcock (2004) the first author presents interview data in which mathematicians stress the importance of these skills.

We therefore wish to recognize the value in what each student is doing, to think in terms of helping students build upon their existing strategies, and to augment these with skills they may possess but tend not to invoke without prompting. This seems eminently possible in the cases of relatively successful students like those examined here. Brad did not think only about examples, he also thought in terms of using standard proof structures. In addition, the examples he introduced were all appropriate, showing that he could correctly process the syntax of both known and given definitions. If he could be encouraged to begin his proof attempts more systematically by clearly writing down assumptions, and to spend some time linking the symbols of the definition with his examples, he might be better able to use his example-based insights to guide his proofs. Similarly, Carla did not only think about syntax, she also reported that she used examples to aid her in understanding the statements and proofs she was given in the course. In addition, when asked specifically to illustrate a given proof with examples, she was able to do so correctly and without much apparent difficulty. If she could be encouraged to make 
this activity a more regular part of her work, she might gain a more consistent sense of being able to "imagine what is going on," more fluency in constructing counterexamples, and perhaps more insight into how to translate from examples to properties as well as the reverse.

Of course, in a classroom setting, one is likely to have to deal simultaneously with students with different combinations of available strategies and skills. Encouragingly, we can observe a commonality in the difficulties encountered by both Brad and Carla: each seemed to have an underdeveloped notion of how to use examples and syntax together to construct a proof. Hence, we suggest that in class settings students with both syntactic and referential preferences might benefit from instruction that explicitly draws attention to links between formal statements and proofs and their referent objects and relationships, at a detailed, step-by-step level. This might help students to see more clearly how different ways of interacting with mathematical statements can be complementary and to make informed selections from a wider range of strategies than they might naturally employ.

\section{References}

Alcock, L. J. (2004). Uses of example objects in proving. In M. Johnson Hines \& A. B. Fuglestad (Eds.), Proceedings of the 28th annual conference of the International Group for the Psychology of Mathematics Education, Vol. 2 (pp. 1724). Bergen, Norway: Bergen University College.

Alcock, L. J. (2008). Mathematicians' perspectives on the teaching and learning of proof. In F. Hitt, D. A. Holton, \& P. Thompson (Eds.), Research in collegiate mathematics education. VII (pp. 73-100). Providence, RI: American Mathematical Society.

Alcock, L. J., \& Simpson A. P. (2002). Definitions: Dealing with categories mathematically. For the Learning of Mathematics, 22(2), 28-34.

Alcock, L. J., \& Simpson A. P. (2004). Convergence of sequences and series: Interactions between visual reasoning and the learner's beliefs about their own role. Educational Studies in Mathematics, 57, 1-32.

Alcock, L. J., \& Simpson A. P. (2005). Convergence of sequences and series 2: Interactions between non-visual reasoning and the learner's beliefs about their own role. Educational Studies in Mathematics, 58, 77-100.

Dubinsky, E., Elterman, F., \& Gong, C. (1988). The student's construction of quantification. For the Learning of Mathematics, 8(2), 44-51.

Dubinsky, E., \& Yiparaki, O. (2000). On student understanding of AE and EA quantification. In E. Dubinsky, A. H. Schoenfeld, \& J. Kaput (Eds.), Research in collegiate mathematics education. IV (pp. 239-289). Providence, RI: American Mathematical Society.

Fischbein, E. (1982). Intuition and proof. For the Learning of Mathematics, 3(2), 9-24.

Griffiths, P. A. (2000). Mathematics at the turn of the millennium. American Mathematical Monthly, 10\%, 1-14.

Hanna, G. (1991). Mathematical proof. In D. O. Tall (Ed.), Advanced mathematical thinking (pp. 54-61). Dordrecht: Kluwer.

Harel, G., \& Sowder, L. (1998). Students' proof schemes: Results from exploratory studies. In A. H. Schoenfeld, J. Kaput, \& E. Dubinsky (Eds.), Research in 
collegiate mathematics education. III (pp. 234-283). Providence, RI: American Mathematical Society.

Harel, G. \& Sowder, L. (2007). Toward comprehensive perspectives on the teaching and learning of proof. In F. Lester (Ed.), Second handbook of research on mathematics teaching and learning (pp. 805-842). Charlotte, NC: Information Age.

Inglis, M., Mejia-Ramos, J. P., \& Simpson, A. (2007). Modelling mathematical argumentation: The importance of qualification. Educational Studies in Mathematics, 66, 3-21.

Lakoff, G. \& Núñez, R. E. (2000). Where mathematics comes from. New York: Basic Books.

Moore, R.C. (1994). Making the transition to formal proof. Educational Studies in Mathematics, 27, 249-266.

Pinto, M.. \& Tall, D. O. (1999). Student construction of formal theories: Giving and extracting meaning. In O. Zaslavksy (Ed.), Proceedings of the 23rd annual conference for the International Group on the Psychology of Mathematics Education, Vol.1 (pp. 281-288). Haifa, Israel: Technion - Israel Institute of Technology.

Pinto, M.. \& Tall, D. O. (2002). Building formal mathematics on visual imagery: A case study and a theory. For the Learning of Mathematics, 22(1), 2-10.

Raman, M. (2003). Key ideas: What are they and how can they help us understand how people view proof? Educational Studies in Mathematics, 52, 319-325.

Recio, A. M. \& Godino, J. D. (2001). Institutional and personal meanings of mathematical proof. Educational Studies in Mathematics, 48, 83-99.

Schoenfeld, A. H. (1985). Mathematical problem solving, Orlando, FL: Academic.

Selden, A. \& Selden, J. (2007). Overcoming students' difficulties in learning to understand and construct proofs. (Tech. Rep. No. 2007-1). Cookeville, TN: Tennessee Technological University, Department of Mathematics.

Selden, A., \& Selden, J. (2003). Validation of proofs considered as texts: Can undergraduates tell whether an argument proves a theorem? Journal for Research in Mathematics Education, 34, 4-36.

Selden, J., \& Selden, A. (1995). Unpacking the logic of mathematical statements. Educational Studies in Mathematics, 29, 123-151.

Sfard, A. (1991). On the dual nature of mathematical conceptions: Reflections on processes and objects as different sides of the same coin. Educational Studies in Mathematics, 22, 1-36.

Tall, D. O. (1997). Metaphorical objects in advanced mathematical thinking. International Journal for Computers in Mathematics Learning, 1, 61-65.

Thurston, W. P. (1994). On proof and progress in mathematics. Bulletin of the American Mathematical Society, 30, 161-177.

Vinner, S. (1991). The role of definitions in teaching and learning mathematics. In D. O. Tall (Ed.), Advanced mathematical thinking (pp. 65-81), Dordrecht: Kluwer.

Weber, K. (2001). Student difficulty in constructing proofs: The need for strategic knowledge. Educational Studies in Mathematics, 48, 101-119.

Weber, K. (2003). Students' difficulties with proof. In A. Selden \& J. Selden (Eds.), Research Sampler, 8. Retrieved June 17, 2006 from http://www.maa.org/t_and_l/sampler/rs_8.html. 
Weber, K., \& Alcock, L. J. (2004). Syntactic and semantic production of proofs. Educational Studies in Mathematics, 56, 209-234.

Weber, K., Alcock, L. J., \& Radu, I. (2005). Undergraduates' use of examples in a transition to proof course. Proceedings of the 27th annual conference of the North American Chapter of the International Group for the Psychology of Mathematics Education. Roanoake, VA: Virginia Polytechnic Institute and State University. 


\section{Appendix: Proof for Proof-Reading Task}

Proof (by cases)

Assume $f$ and $g$ are increasing functions and $f(0)=g(0)=0$.

Let $x \in \mathbf{R}$.

By the law of trichotomy, either $x>0, x<0$, or $x=0$.

Case 1. Suppose $x>0$.

Since $x>0$ and $f$ is increasing, $f(x)>f(0)$ by definition of increasing function. Since $f(0)=0, f(x)>0$.

Since $x>0$ and $g$ is increasing, $g(x)>g(0)$ by definition of increasing function. Since $g(0)=0, g(x)>0$.

Since $f(x)$ and $g(x)$ are positive, $f(x) \cdot g(x)$ is positive and hence $f(x) \cdot g(x) \geq 0$.

Case 2. Suppose $x<0$.

Since $x<0$ and $f$ is increasing, $f(x)<f(0)$ by definition of increasing function. Since $f(0)=0, f(x)<0$.

Since $x<0$ and $g$ is increasing, $g(x)<g(0)$ by definition of increasing function. Since $g(0)=0, g(x)<0$.

Since $f(x)$ and $g(x)$ are negative, $f(x) \cdot g(x)$ is positive and hence $f(x) \cdot g(x) \geq 0$.

Case 3. Suppose $x=0$.

Then $f(x) \cdot g(x)=f(0) \cdot g(0)=0 \cdot 0=0$.

So $f(x) \cdot g(x) \geq 0$.

Since in all three cases, $f(x) \cdot g(x) \geq 0, \forall x \in \mathbf{R}(f(x) \cdot g(x) \geq 0)$.

Department of Mathematical Sciences, University of Essex, Wivenhoe Park, ColCHESTER, EsSEX, CO4 3SQ, UK

E-mail address: lalcock@essex.ac.uk

Graduate School of Education, Rutgers University, 10 Seminary Place, New BrunsWICK, NJ 08901, USA

E-mail address: khweber@rci.rutgers.edu 